\title{
La participation sociale dans une communauté rurale au Québec : ce qui la facilite, selon les points de vue de personnes engagées
}

\author{
Marc-André Bonneau ${ }^{a}$, Sophie Dupéréb
}

RÉSUMÉ. Cet article a pour objectif de présenter et de discuter certains facteurs facilitant la participation sociale, selon la perspective de personnes donnant de leur temps dans une petite communauté rurale. Cette implication est particulièrement importante pour les communautés situées dans une région dévitalisée sur les plans social et économique. Les résultats découlent d'une recherche qualitative effectuée auprès de 12 personnes engagées dans une communauté rurale située dans la région du Centre-du-Québec, au Québec. L'analyse thématique a fait ressortir six éléments facilitant la participation : 1) le besoin de revitaliser la communauté, 2) la présence de soutien municipal, 3) le dynamisme lié à la présence de nouveaux arrivants, 4) un climat de confiance, de réciprocité et d'entraide, 5) l'utilisation des réseaux sociaux et 6) un désir d'autonomie collective. À partir de ces résultats, nous discutons de l'importance de soutenir la participation sociale pour le développement socioéconomique de communautés rurales et pour la réduction des inégalités sociales.

\begin{abstract}
This article presents and discusses some factors facilitating social participation in a small rural community for people volunteering their time. This involvement is particularly important for communities located in a socially and economically devitalized region. The qualitative results present the thematic analysis of 12 interviews conducted in a rural community located in the Centre-du-Québec region of Quebec. The analysis identified six contextual elements facilitating participation: 1) the need to revitalize the community, 2) the presence of municipal support, 3) the dynamism related to the presence of newcomers, 4) a climate of trust, reciprocity and mutual support, 5) the use of social networks, and 6) a desire for collective autonomy. Based on these results, we discuss the importance of supporting social participation for the socio-economic development of rural communities and the reduction of social inequalities.
\end{abstract}

\section{Introduction et mise en contexte}

Au Québec, l'implication des personnes dans leur milieu a toujours occupé une place centrale dans le développement des communautés rurales. Ce travail, qui demeure souvent invisible, alimente l'innovation et le changement social, enrichit les liens sociaux et la résilience communautaire, et améliore la qualité de vie (Birtch, 2017; Malet, 2009; Reimer, 2006). Il est d'une grande importance pour le développement des petites communautés rurales et pour le fonctionnement des organisations qui animent le milieu. D'ailleurs, la participation à la vie communautaire est plus présente dans les régions

\footnotetext{
a Diplômé, maittrise en santé communautaire, Université Laval

${ }^{\mathrm{b}}$ Professeure agrégée, Faculté des sciences infirmières, Université Laval
}

rurales que dans les grands centres au Québec, selon la dernière analyse disponible de l'Enquête sociale générale du Canada (Statistique Canada, 2004; Turcotte, 2005).

En 2012, plus du tiers des Québécois a fait un travail bénévole (Gravel, 2017). Ces derniers se distinguent de l'ensemble des Canadiens en s'impliquant légèrement moins et en réalisant un travail bénévole plus informel, à l'extérieur d'organisations (Gravel, 2017). Cependant, le nombre total d'heures de bénévolat a diminué au Québec, passant de 308 millions d'heures annuelles à 267 millions d'heures entre 2004 et 2013 (Gravel, 2017). 
Considérant que l'implication bénévole des personnes est centrale pour le développement social et économique des communautés rurales (Klein, 2012; Reimer, 2006; Tremblay et Klein, 1997), il est important de mieux comprendre les facteurs contextuels qui ne se limitent pas à la motivation d'une seule personne et qui facilitent cette forme de participation sociale. Trop souvent, une minorité de bénévoles (" toujours les mêmes ») effectue une grande part des heures de travail. Cela soulève l'importance de mobiliser d'autres citoyens pour mettre la main à la pâte, particulièrement en ruralité, où les bénévoles sont souvent assez âgés (Turcotte, 2005). C'est dans ce contexte que le présent article a pour objectif de présenter et de discuter les facteurs contextuels facilitant la participation sociale, selon les perspectives de personnes engagées dans une petite communauté rurale.

L'implication bénévole a été conceptualisée dans notre projet comme une forme de participation sociale, c'est-à-dire «l'action de contribuer à la collectivité en donnant du temps aux organisations et aux individus à l'extérieur de la sphère domestique » Gaudet, 2012, p. 2). Cette définition s'appuie sur les travaux sur le don du sociologue québécois Jacques T. Godbout, pour qui la participation sociale se distingue de la relation marchande par la gratuité qui la caractérise (Godbout, 1992). Godbout souligne « la nécessité de comprendre d'autres formes de logiques d'action que celle de l'intérêt» (Gaudet et Turcotte, 2013, p. 119), comme celles des affects et de la construction identitaire.

Le concept de participation sociale regroupe plusieurs pratiques, qui tendent à être ignorées par manque de reconnaissance institutionnelle (Gaudet, 2012). Parmi celles-ci, Gaudet identifie des pratiques formelles, par exemple la participation communautaire bénévole et associative (comités citoyens, organisations sans but lucratif), ainsi que des pratiques informelles qui «tissent la solidarité au quotidien » (Gaudet, 2012, p. 2), par exemple l'entraide et le soutien auprès des proches. Le don de temps s'inscrit dans un contexte socioéconomique qui définit les occasions de participation, notamment par le temps disponible pour participer à la vie communautaire ou par la présence de communautés d'intérêts (Gaudet, 2012).

Dans le contexte rural québécois, les facilitateurs contextuels favorisant la participation ont été peu documentés. Ce sont davantage les motivations des individus qui ont été mises de l'avant, comme le désir de contribuer à la communauté ou de mettre à profit ses compétences, notamment par l'Enquête sociale générale du Canada (Vézina et Compton, 2012). Dans la littérature universitaire, la participation a surtout été étudiée à l'intérieur de contextes formels ou d'institutions comme celles du système de santé, particulièrement dans les milieux urbains (Kilpatrick, 2009; Kenny et collab., 2013).

Les milieux ruraux sont souvent perçus comme authentiques et chaleureux, l'engagement des personnes dans leur communauté y étant plus élevé (Jean, 2011). Une analyse réalisée à partir de l'Enquête sociale générale du Canada a fait ressortir que les ruraux sont plus susceptibles d'accomplir un travail non rémunéré et d'éprouver un fort sentiment d'appartenance à l'égard de leur communauté (Statistique Canada, 2004; Turcotte, 2005). Les résultats de cette analyse suggèrent également que la différence entre la participation dans les milieux ruraux et urbains ne s'explique pas simplement à cause des caractéristiques socioéconomiques différentes entre les personnes, comme le revenu, mais davantage par des caractéristiques contextuelles, comme la connaissance de ses voisins (Turcotte, 2005; Putnam, 2000).

La littérature sur le sujet offre plusieurs pistes pour expliquer l'influence de la ruralité sur la participation. Par exemple, en comparaison avec les centres urbains, les populations rurales s'impliqueraient davantage grâce à leur homogénéité sociale et à la faible mobilité des personnes (Turcotte, 2005). Ces facteurs faciliteraient l'émergence de normes sociales qui encouragent la participation (Gaudet et Turcotte, 2013). Également, la présence d'un sentiment d'appartenance plus élevé dans les petites communautés rurales pourrait également faciliter l'émergence de telles normes (Falk et Kilpatrick, 2000).

La participation dans les communautés rurales québécoises revêt une importance particulière en raison des transformations sociales et économiques auxquelles elles font face depuis les dernières décennies. Ces transformations ont été abordées comme un phénomène de "dévitalisation» par le ministère des Affaires municipales, des Régions et de l'Occupation du territoire (MAMROT, 2013). Ce phénomène a notamment été caractérisé par un important déclin démographique, à la suite de 
l'exode vers les milieux urbains et en raison du vieillissement de la population, ainsi que par la perte de services de proximité (aliments, essence, etc.).

La dévitalisation est un phénomène cyclique, auquel s'ajoutent d'autres réalités, dont le faible niveau de scolarité de ses habitants, une perte de fierté et d'appartenance ainsi que le manque d'emploi (Groupe de travail sur les communautés dévitalisées, 2010). À l'échelle globale, cette problématique s'inscrit dans un contexte de libre-échange et de désengagement de l'État (Reimer, 2006; Skinner et Joseph, 2011). Au Québec, ces transformations ont été étudiées à travers différentes perspectives, dont le développement social (Bourque et Favreau, 2003) et le développement territorial (Polèse, 2018; Parent, Lachapelle, Bourque et Jetté, 2016).

Afin de briser ce cycle et d'atténuer ces transformations, plusieurs chercheurs et décideurs ont mis de l'avant l'hypothèse selon laquelle la participation devient un incontournable. Ce constat a été fait notamment en raison de l'incapacité des gouvernements à résoudre cette problématique, notamment parce que les solutions proposées provenaient très peu des communautés touchées par ces transformations (Boisvert, 2013; Lachapelle et Bourque, 2008). À ce sujet, le rapport du Groupe de travail sur les communautés dévitalisées, mis sur pied par le MAMROT, a conclu que « la mobilisation de la population et l'engagement bénévole sont des éléments déterminants dans la revitalisation» (2010, p. 32).

Dans le même ordre d'idées, plusieurs ont reconnu et rappelé l'importance de la participation pour le développement à l'échelle locale (Boisvert, 2013; Lachapelle et Bourque, 2008; Lévesque, Delisle et Leroux, 2002). Parmi ceux-ci, Boisvert (2013) rappelle que les initiatives locales soutenues par la participation sont souvent plus efficaces puisqu'elles permettent un développement des capacités des premiers concernés, ce qui peut aussi par la bande contribuer à réduire les inégalités sociales. La participation «est un passage obligé pour que les milieux puissent prendre en charge le développement de leur communauté » (Lévesque, Delisle et Leroux, 2002, p. 6, cités dans Lachapelle et Bourque, 2008, p. 74).

Malgré ces constats, plusieurs mesures visant à soutenir le développement rural et la participation locale ont été abolies en 2014 et 2015 par le gouvernement libéral de l'époque. La plus importante de celles-ci était la Politique nationale de la ruralité (PNR), qui permettait notamment de soutenir le développement rural à travers des ententes entre le gouvernement du Québec, les municipalités régionales de comté (MRC) et les municipalités rurales.

En 2014, le gouvernement du Québec a également retiré son financement à l'organisme Solidarité rurale du Québec, qui agissait à titre d'instanceconseil. Cet organisme avait pour mission de «promouvoir la revitalisation et le développement du monde rural [...] de manière à renverser le mouvement de déclin et de déstructuration des campagnes québécoises » (Solidarité rurale du Québec, 2011, p. 4).

En 2015, par le biais de la Loi concernant principalement la mise en cuure de certaines dispositions du discours sur le budget du 4 juin 2014 et visant le retour à l'équilibre budgétaire en 2015-2016 (Québec, 2015), le gouvernement du Québec a finalement retiré son financement à différents organismes de développement régional tels les Conférences régionales des élus (CRE) et les Centres locaux de développement (CLD). Même si, en 2017, le Fonds d'appui au rayonnement des régions a été annoncé afin de soutenir leur développement, ce fonds a été géré à l'échelle régionale, et non plus remis directement à certaines municipalités dévitalisées, comme c'était le cas auparavant avec la PNR.

Cette nouvelle gouvernance amène ainsi une «perte d'ancrages institutionnels » (Richard, Bourque et Lachapelle, 2017, p. 11), qui semble fragiliser les espaces de participation (Alberio, 2015). En somme, les politiques d'austérité mises en place rendent la participation sociale en ruralité encore plus nécessaire, tout en la rendant plus difficile, compte tenu d'un manque criant de ressources pour appuyer les initiatives locales (Skinner et Joseph, 2011; Klein, 2012; Boisvert, 2013). De plus, tous ces bouleversements s'effectuent dans un contexte où les municipalités québécoises se sont vu confier de plus en plus de responsabilités à l'égard du bien-être dans leur milieu (Scalzo, 2016; FQM, 2015). Ce nouveau contexte de la ruralité met donc en lumière l'importance d'identifier les facteurs contextuels facilitant la participation à l'échelle locale, ce à quoi notre recherche a contribué. 


\section{Méthodologie et milieu de l'étude}

Cet article est l'aboutissement d'un projet réalisé dans le cadre d'une maitrise en santé communautaire à l'Université Laval (Bonneau, 2018). Le milieu à l'étude est une petite communauté rurale caractérisée par une grande implication des personnes dans leur milieu. Celle-ci compte moins de 1000 habitants et est située à moins de $100 \mathrm{~km}$ d'un centre urbain important. Au début des années 2000, elle était l'une des plus dévitalisées du Centre-du-Québec. Dans les dernières décennies, cette communauté a dû composer avec un déclin démographique important et une perte de services de proximité. Cette situation a engendré une prise en main et une forte mobilisation par des citoyens souhaitant redynamiser leur milieu de vie. En ayant le statut de municipalité dévitalisée, cette petite communauté a bénéficié de financement qui a soutenu sa revitalisation, notamment par le biais de la PNR. Cette politique a permis l'embauche d'une personne chargée de projet ainsi que l'amélioration d'infrastructures municipales (maison des jeunes, parc, etc.). Le nom de la municipalité étudiée est gardé confidentiel pour faciliter le respect de l'anonymat de chaque participant.

Au total, 12 personnes ont été interviewées à l'aide d'entretiens semi-dirigés, de manière à présenter une diversité de formes de participation sociale. Le nombre de participants s'explique par la petite taille de la communauté à l'étude et par un effort d'atteindre une saturation théorique des données (Gohier, 2004). Pour participer à cette recherche, les personnes devaient avoir plus de 18 ans et donner du temps à leur communauté à l'extérieur du contexte familial. À titre d'exemples, une personne interrogée était impliquée dans un comité de loisirs, tandis qu'une autre aidait à l'organisation d'évènements pour financer un organisme sans but lucratif de la communauté.

Ces personnes ont été invitées à décrire leur participation ainsi que les retombées sur la santé et le bien-être tant d'elles-mêmes que de leur communauté (Bonneau, 2018). L'approche suivie s'est inspirée des récits de vie telle qu'elle a été formulée par Bertaux (2016). À la suite d'une analyse thématique réalisée à l'aide du logiciel d'analyse qualitative NVivo, les entretiens ont également permis de documenter plusieurs éléments contextuels facilitant la participation des personnes interrogées.

\section{Résultats \\ 2.1 Les types de participation documentés}

Les personnes interviewées sont engagées dans divers types de participation sociale volontaire et non rémunérée dans leur milieu. Pour reprendre la typologie de Gaudet (2012), nous distinguons deux types de participation, qui se retrouvent dans les expériences documentées.

D'abord, les pratiques formelles (militance, participation politique, communautaire et associative au sein d'une organisation), puis les pratiques informelles (entraide, soutien et soins offerts à l'extérieur de la sphère domestique). L'ensemble des personnes rencontrées indique pratiquer des activités informelles ainsi que des activités formelles. Les participants étaient invités à décrire leur participation sociale ainsi que ses retombées. Certains décrivent une participation occasionnelle, tandis que d'autres rapportent une participation régulière et assidue. Toutes les personnes interviewées soutiennent que leur implication formelle s'est échelonnée sur plusieurs années. Près de la moitié des répondants sont impliqués depuis près de 10 ans dans leur milieu. Plusieurs mentionnent que leur implication occupe donc une place significative dans leur vie.

Le tableau 1 présente les principales caractéristiques des participants et de leur participation. Parmi les pratiques informelles recensées, on retrouve divers exemples d'entraide et de soutien. Ces actions sont principalement de nature provisoire et s'étalent sur des périodes relativement courtes (de quelques jours à quelques semaines). Plusieurs répondants mentionnent des échanges de services entre les personnes de la communauté ou de soutien à l'endroit de personnes défavorisées. Par exemple, un répondant évoque le soutien qu'il a apporté à l'un de ses voisins souffrant d'une dépression. Un autre met de l'avant l'aide apportée par ses voisins après qu'il eut eu un accident de voiture. Ceux-ci allaient chercher des médicaments pour le répondant, ce qu'il n'était pas en mesure de faire pendant quelques semaines. 


\begin{tabular}{|l|c|c|l|l|}
\hline Participant & Genre & $\begin{array}{c}\text { Groupe } \\
\text { d'âge }\end{array}$ & Occupation & $\begin{array}{l}\text { Participation principale de la } \\
\text { personne interviewée }\end{array}$ \\
\hline 1 & H & 46 à 55 & Artiste & Informelle (évènement) \\
\hline 2 & F & 36 à 45 & Artiste & Informelle (évènement) \\
\hline 3 & F & 76 et plus & Retraitée & Formelle (comité) \\
\hline 4 & F & 36 à 45 & Salariée & Informelle (évènement) \\
\hline 5 & F & 36 à 45 & Salariée & Formelle (comité) \\
\hline 6 & H & 56 à 75 & Travailleur autonome & Formelle (comité) \\
\hline 7 & F & 18 à 35 & Étudiante & Informelle (évènement) \\
\hline 8 & H & 56 à 75 & Sans emploi & Informelle (évènement) \\
\hline 9 & F & 36 à 45 & Salariée & Formelle (conseil d'administration) \\
\hline 10 & F & 56 à 75 & Retraitée & Formelle (conseil d'administration) \\
\hline 11 & H & 56 à 75 & Retraité & Formelle (conseil d'administration) \\
\hline 12 & H & 18 à 35 & Salarié & Informelle (évènement) \\
\hline
\end{tabular}

Tableau 1 - Description des participants

Parmi les pratiques formelles évoquées, divers exemples de don de temps qui prennent place à l'intérieur d'organisations sont présentés. Malgré sa petite taille, la communauté en regroupe plusieurs, dont une coopérative de solidarité qui gère une station-service et un casse-croûte, une association mettant sur pied des activités récréotouristiques équestres et un organisme citoyen qui vise notamment la protection et la mise en valeur des milieux naturels. À ces organismes s'ajoutent des comités citoyens variés, par exemple un comité de développement, un comité récréatif et un comité arboricole. Également, des regroupements plus traditionnels comme le Conseil de fabrique ou le Cercle des fermières sont présents dans la communauté. C'est à l'intérieur de ces organismes et de ces comités que les pratiques de participation formelle se sont déployées. Rappelons aussi qu'au sein d'une même organisation, la participation sociale peut prendre différentes formes, par exemple une contribution ponctuelle lors d'un évènement ou une implication assidue au conseil d'administration.

\subsection{Les facteurs contextuels facilitant la participation}

L'analyse des propos recueillis a fait ressortir six facteurs contextuels facilitant la participation : 1) le besoin de revitaliser la communauté, 2) la présence de soutien municipal, 3) le dynamisme lié à la présence de jeunes familles et de nouveaux arrivants, 4) un climat de confiance, de réciprocité et d'entraide, 5) l'utilisation des réseaux sociaux et 6) un désir d'autonomie collective.

\subsubsection{Le besoin de revitaliser la communauté}

La participation à l'intérieur de cette municipalité apparait grandement influencée par la prise de conscience, quelques années auparavant, du fait que c'était une des communautés les plus dévitalisées de la région. L'effort collectif de revitalisation a fait suite à ce constat. Cet effort de redynamisation a eu un impact considérable sur la cohésion du milieu et sur la motivation des participants à s'y investir. Certains organismes, tels que la coopérative de solidarité, ont d'ailleurs pris naissance dans ce contexte afin de répondre au besoin vital de maintenir la pérennité des services de proximité.

Comme l'explique une répondante: «Les petites paroisses, aujourd'hui, s'il n'y a pas de bénévolat, ça ne peut pas fonctionner. » Ainsi, pour plusieurs des personnes interviewées, le maintien de certains services est conditionnel à l'implication des citoyens dans leur milieu. Ce motif est particulièrement visible auprès des jeunes mères de famille qui s'impliquent pour favoriser l'accès aux loisirs dans la communauté. De plus, des participants signalent avoir choisi de s'établir dans cette municipalité en raison de cet effort collectif pour revitaliser le milieu. Une des participantes explique ainsi sa décision : «Je suis devenue propriétaire ici, en me disant [...] il y a pleins d'actions pour remonter la vitalité. »

Plusieurs des répondants présentent cet effort collectif comme un facteur favorisant leur propre implication. De plus, pour une bénévole qui travaille également pour la municipalité, la présence d'une vie communautaire est essentielle pour la rétention des résidents : «Sans implication, tu vas peut-être finir 
par te sentir isolé. Tu vas avoir envie de quitter. Moi, je pense que c'est encore plus fort dans les petites communautés. » Cet enjeu est crucial pour assurer un certain dynamisme et pour appuyer le développement du milieu : "C'est comme essentiel. Le monde rural ne serait pas viable sans la participation des gens. On n'a pas les ressources matérielles ou financières. » Pour une autre personne interviewée, cette réalité a permis d'aller chercher des gens qui ne seraient pas impliqués autrement.

\subsubsection{Le soutien matériel et organsationnel de la municipalité}

Près du tiers des participants met de l'avant le constat selon lequel la participation est facilitée par le soutien matériel et organisationnel qu'offre la municipalité aux citoyens qui désirent mettre de l'avant des projets. À ce sujet, près de la moitié des répondants ont évoqué l'importance du travail effectué par les personnes chargées de projet travaillant pour la municipalité. Celles-ci soutiennent des comités et des associations, notamment pour l'organisation d'évènements et pour la recherche de financement. De manière générale, leurs liens avec les personnes impliquées semblent également contribuer au maintien de l'engagement social. Le travail de ces employés municipaux contribue également à la reconnaissance des personnes qui s'impliquent, à travers le journal local mensuel ou en organisant des activités comme le gala des bénévoles.

Comme l'explique une répondante, les chargés de projet exercent du leadership qui permet de soutenir la participation sociale dans la communauté : «Ces deux personnes ont trouvé un salaire de peut-être deux ans... Le bénévolat, ça fonctionne quand il y a une tête qui essaie de diriger tout ça.» Ainsi, ces citoyens engagés contribuent grandement au dynamisme du milieu.

Un bon accès aux élus municipaux apparait également comme une autre forme de soutien municipal pour les personnes qui s'impliquent bénévolement. Trois répondants mettent de l'avant le fait que les élus sont plus accessibles dans la municipalité à l'étude, en comparaison avec d'autres municipalités avoisinantes ou avec des centres urbains. Selon ces répondants, cette proximité bénéficierait aux initiatives citoyennes et faciliterait la participation sociale. L'un d'eux illustre cette proximité en décrivant l'écoute qui caractérise l'appareil municipal : « La mairesse, les gens du village, ils nous sont compréhensifs. Ils nous donnent la chance. On a un contact humain. Ce n'est pas une grosse machine qui dirige tout le monde.»

De plus, quelques répondants soulignent également que, dans le passé, la présence de programmes de financement appuyant spécifiquement les municipalités rurales dévitalisées a permis de soutenir la participation. Par exemple, dans la communauté à l'étude, des programmes provinciaux de financement ont permis l'embauche de chargés de projet qui ont largement animé la participation des citoyens dans leur milieu : «Avant que les chargés de projet arrivent, il ne se passait plus rien ici. Rien. » Selon une personne interviewée, ces programmes sont particulièrement importants puisque les petites municipalités ne possèdent pas les ressources pour les embaucher : «Les chargés de projet, c'est eux qui cherchent leur paye. La municipalité ne serait pas capable de payer leur salaire. Ils vont chercher les subventions. Autrement, ils ne seraient pas là. »

\subsubsection{Un climat de confiance, de réciprocité et d'entraide}

La participation semble aussi renforcée par la cohésion sociale du milieu, qui a été identifiée par plusieurs participants comme une caractéristique des milieux ruraux. La communauté est "tissée serré", affirme une jeune répondante qui aide lors d'évènements organisés par le Cercle des fermières. Plusieurs mettent de l'avant lidée selon laquelle la petite taille du milieu modifie les relations sociales et que celles-ci sont caractérisées par une plus grande proximité entre les citoyens.

Une répondante explique que, malgré les grandes distances entre les maisons et la présence de rangs éloignés, la taille de la communauté contribue à créer un certain filet social : "Un feeling que OK, peut-être que, quand je suis à la maison, toute seule, je peux feeler toute seule au monde. Mais, si je sors, il y a des échanges. Ça contribue à ce qu'on ne se sente pas anonyme. »

Dans le même ordre d'idées, un autre répondant apprécie le fait de souvent recroiser les mêmes personnes lors de ses implications, ce qui est lié à la petite taille du milieu. Dans ce contexte, il explique qu'il est plus porté à participer à des activités dans le village : "C'est le fun de revoir le même monde ici. Puis là, je sors au village et je ne suis pas gêné d'y aller tout seul. » 
Le peu d'emplois dans la communauté pourrait contribuer à ce climat d'entraide en faisant du bénévolat une bonne manière de préserver les liens sociaux : «En faisant du bénévolat, c’est une belle façon. Tu ne peux pas t'impliquer dans du travail ici, car il n'y en a presque pas. »

Dans le même ordre d'idées, d'autres répondants décrivent la présence de réseaux d'entraide informels, qu'ils jugent incomparables à ceux des milieux urbains. Par exemple, un répondant indique notamment comment, lors d'épisodes de maladie, plusieurs voisins lui ont apporté du soutien de manière spontanée: "Je pense que le fait que ça soit plus petit, le fait que les ressources ne soient pas toujours à côté, il y a peut-être un peu plus de coopération. C'est comme si les gens s'enfargeaient un peu moins dans les fleurs du tapis. » Un autre participant soutient que le caractère informel de l'entraide permet d'apporter de l'aide sous une forme plus humaine, qui correspond davantage aux besoins des personnes du milieu. La présence de formes d'entraide et de réciprocité particulières aux milieux ruraux facilite donc la participation sociale des citoyens.

\subsubsection{Le dynamisme lié à la présence de jeunes familles et de nouveaux arrivants}

Plusieurs participants soulignent que la présence de jeunes familles souhaitant s'impliquer pour obtenir des services contribue également au dynamisme du milieu. De jeunes mères de famille ont remarqué la proportion particulièrement élevée d'enfants par rapport au nombre total d'habitants de la municipalité, ce qui fait notamment suite à l'arrivée de nouvelles familles dans la communauté. Des répondants expliquent que le faible coût des propriétés a contribué entre autres à l'arrivée de familles, ce qui a dynamisé l'implication des citoyens dans leur milieu. Cette présence a été identifiée comme un facteur encourageant la participation puisque ces jeunes familles souhaitent améliorer les services disponibles.

Le souhait que la communauté se développe et bonifie ses services à long terme est une considération qui alimente la participation chez certains répondants : «C'est un héritage qu'on donne aux générations qui vont nous suivre", affirme un participant âgé. En parlant des nouveaux arrivants, ce dernier fait le lien avec les projets actuels et les retombées futures: "C'est en montant des projets comme ceux qu'ils montent que ça va aller chercher l'environnement pour leurs enfants. C'est important pour eux. » Ainsi, l'implication est également causée par une préoccupation d'améliorer les services qu'offre la communauté et d'assurer sa pérennité.

\subsubsection{L'utilisation des réseaux sociaux qui soutiennent la participation}

Quelques participants mettent également de l'avant l'idée selon laquelle la présence de réseaux de communication permet d'informer les personnes vivant dans la communauté des besoins éprouvés, ce qui facilite l'implication. Par exemple, deux répondants soulignent que leur don de temps est facilité par l'utilisation des réseaux sociaux. La communauté détient une page Facebook, qui compte autant de membres que de résidents. Une artiste impliquée souligne que cette page, jumelée à la petite taille du milieu, permet une grande solidarité entre voisins : « [En ville,] il n’y aurait pas eu cet échange-là. On revit beaucoup le troc. » Ce média numérique est particulièrement important, selon cette répondante, dans un contexte où il y a peu de services formels à proximité.

Ainsi, selon quelques personnes interviewées, ces plateformes de communication encouragent et facilitent la participation sociale. Les liens sociaux apparaissent influencés par la petite taille de la communauté : «Oui, il y a des bénévoles qui s'impliquent aussi. Je pense à la ville voisine quand elle fait de grands évènements, mais c'est une grosse machine. Les gens se sentent moins interpellés. » Quelques répondants âgés expliquent qu'avant de prendre leur retraite, ils ne connaissaient pas les besoins des organismes du milieu. Toutefois, les réseaux d'entraide en place les ont amenés à mieux comprendre les besoins et à s'impliquer davantage.

Ce facilitateur rejoint également les propos d'une autre participante, qui soulève que les communautés rurales ont des besoins particuliers, mieux compris par ceux qui sont impliqués dans le milieu.

\subsubsection{Un désir partagé d'autonomie collective}

La préoccupation de répondre aux besoins du milieu s'inscrit également dans un désir d'autonomie collective puisque des personnes interviewées 
soulignent qu'elles apprécient que leur communauté puisse répondre à ses besoins sans aide ni intervention extérieures. Le désir que leur communauté demeure autonome en maintenant une vie communautaire dynamique et en soutenant le développement économique contribue à la participation des citoyens à l'intérieur du milieu.

Une répondante impliquée illustre ainsi cette réalité : «Il y a ça de beau, dans les milieux ruraux : on a la peur des grosses organisations qui viennent de la ville, qui viennent te dire comment faire et qui viennent d'ailleurs. Mais qu'on soit capable en tant que société de s'aider entre nous... Ça aussi, c'est un plus. »

Cette préoccupation pour l'autonomie du milieu facilite la participation, dans le sens où celle-ci apparaît comme un moyen incontournable pour répondre aux besoins spécifiques. Ainsi, le don de temps a également une fonction symbolique, qui valorise le milieu : «Ça parle en bien de nous et de la place, ce qui nous donne une grande fierté à nous, les bénévoles.» Un autre participant fait l'éloge de l'implication des personnes dans leur milieu en soulignant que les associations sans but lucratif ont permis de créer de l'emploi dans la communauté. Selon lui, ces retombées et les réalisations du passé amènent d'autres citoyens à s'impliquer.

\section{Discussion : soutenir la particpation sociale pour le développement des communautés rurales dans une perspective de réduction des inégalités sociales}

Les résultats de notre recherche qualitative éclairent sur des leviers locaux importants pour développer et soutenir l'engagement des personnes dans les milieux ruraux. Particulièrement chez les acteurs à l'échelle municipale, nos résultats soulèvent l'importance de développer une vision pour l'avenir de la municipalité, d'offrir du soutien aux citoyens qui s'impliquent ainsi que de reconnaître et de valoriser leurs actions.

Dans la municipalité à l'étude, les propos recueillis suggèrent que l'implication bénévole est facilitée et motivée par le désir de dynamiser la communauté dans un contexte de dévitalisation et de faibles ressources. La participation des personnes constitue une manière de faire face aux changements sociaux, économiques et politiques affectant leur communauté. Dans le milieu à l'étude, la participation sociale est liée à une prémisse propre à cette communauté : «Sans bénévolat, on meurt. »

Ces propos font écho à plusieurs constats présents dans la littérature universitaire, où des auteurs relèvent l'importance de la participation dans le contexte rural (Milligan, 2007; Skinner et Joseph, 2011; Reimer, 2006). Pour certaines de ces communautés, dont celle à l'étude, les transformations démographiques, sociales et économiques ont mené à un manque de services et d'occasions auquel la participation peut partiellement répondre. Cette réalité implique des responsabilités grandissantes pour les citoyens impliqués, qui bénéficient de peu de ressources pour soutenir leur action. Par exemple, dans la communauté à l'étude, l'accès à des produits de base (aliments, essence, etc.) à proximité est conditionnel à ce que les citoyens s'impliquent bénévolement pour assurer le fonctionnement de la coopérative de solidarité. Les propos recueillis réaffirment la résilience des communautés rurales face aux transformations globales qui les affectent inévitablement. Cependant, ces citoyens donnent de leur temps en ne bénéficiant que de peu de soutien, ce qui fragilise la pérennité de leurs actions.

En phase avec ce qui est déjà connu, nos résultats sont donc préoccupants pour les communautés qui n'ont pas les ressources ni les capacités de prendre en charge les besoins de leur milieu et de soutenir la participation locale (Jean, 2006; Klein, 2012; Simard, Parent et Richardson, 2018). Ce manque de ressources, en comparaison avec des communautés plus fortunées, pourrait engendrer ou maintenir des inégalités sociales et territoriales (Klein, 2012; Polèse, 2018). Dans ce contexte, la simple participation informelle et non encadrée constitue un «lien ordinaire au politique » (Breviglieri et Gaudet, 2014) puisque, malgré le caractère modeste des actions posées, celles-ci apparaissent fondamentales pour maintenir la présence de services ou d'occasions comparables entre les communautés rurales. Les expériences recueillies montrent l'importance du financement de l'État pour soutenir la participation et ses initiatives, particulièrement pour les communautés en perte de dynamisme. Dans la communauté à l'étude, l'embauche de chargés de projet s'est avérée être déterminante pour soutenir l'implication bénévole. Un tel soutien permet de tendre vers une plus grande équité territoriale (Polèse, 2018) en 
mobilisant les forces locales nécessaires au développement social et économique.

Dans une perspective de réduction des disparités entre les régions québécoises, les nouveaux mécanismes d'attribution et l'abolition de mesures directement destinées aux communautés rurales défavorisées constituent des changements préoccupants. À titre d'exemple, l'impact de l'abolition de la PNR sur les inégalités sociales de santé a déjà été démontré, notamment en relevant les conséquences de son retrait sur les déterminants de la santé à l'échelle locale (Richardson et Simard, 2016). Quelques travaux ont été réalisés pour mieux comprendre l'impact des récentes transformations des politiques publiques sur le développement des territoires (Richard et collab., 2017), mais d'autres études sont nécessaires pour appréhender plus finement les répercussions de ces mesures sur la participation sociale.

Ce contexte est particulièrement préoccupant pour les petites communautés bénéficiant d'un plus faible pouvoir d'action (Klein, 2012; Simard et collab., 2018), qui se retrouvent présentement dans un contexte politique préconisant l'action locale, mais sans l'accompagner de politiques nationales qui favorisent l'équité (Polèse, 2018). Il convient de rappeler ce contexte, car « l'accent mis sur le pouvoir du "local" fait trop facilement abstraction des tendances et contraintes de la géographie économique» (Polèse, 2018, p. 18). Nos résultats réitèrent le potentiel de l'action bénévole locale (Tremblay et Klein, 1997), mais aussi l'importance du soutien des gouvernements municipaux, régionaux et provinciaux.

De plus, pour réduire les inégalités, la force de la participation est aussi celle de favoriser une mixité sociale, notamment pour inclure des personnes en situation de pauvreté ou d'exclusion. Celles-ci peuvent apporter un regard éclairant sur les dynamiques qui engendrent les inégalités (Loignon, Dupéré, Godrie et Leblanc, 2018; Mercier, Bourque et St-Germain, 2009; Richard et collab., 2017).

Ces constats relèvent l'importance de mieux comprendre les facteurs contextuels facilitant la participation et de mener une réflexion plus globale sur le soutien à accorder aux petites communautés rurales «en marge des circuits de la prospérité » (Jean, 2006, p. 469). Il importe de ne pas idéaliser la participation ainsi que de remettre en question le rôle de l'État, sans quoi la participation risque d'être instrumentalisée et, ultimement, ne pas vraiment servir les intérêts locaux (Wallerstein, 2002; Boisvert, 2013). Déjà, plusieurs auteurs ont critiqué le fait que la littérature sur la participation sociale a mis l'accent sur les liens horizontaux entre les personnes, plutôt que sur les rapports de pouvoir verticaux, entre l'État et les communautés locales (Hawe et Shiell, 2000; Wallerstein, 2002). Avec la transformation des mécanismes d'attribution du financement et la nouvelle gouvernance qu'ils impliquent, les résultats présentés s'ajoutent au concert des voix citoyennes qui mettent en valeur l'importance d'avoir une réflexion commune sur le soutien que nous souhaitons collectivement apporter à ces communautés.

\section{Conclusion}

Cet article avait pour objectif de présenter et discuter les facteurs contextuels facilitant la participation sociale en milieu rural dévitalisé, à partir de la perspective de personnes engagées dans une petite communauté rurale québécoise qui, au moment de l'étude, tirait son épingle du jeu. Bien que les résultats présentés ne soient pas généralisables et soient particuliers à une seule communauté, le devis qualitatif de cette étude permet une certaine transférabilité des résultats pour des contextes similaires (Gohier, 2004).

Les résultats offrent des pistes de réflexion intéressantes pour les intervenants et les élus de municipalités rurales qui souhaitent valoriser et soutenir la participation dans leur milieu ainsi que promouvoir le développement social et économique de leur communauté. De plus, la discussion proposée inscrit le rôle de la participation sociale dans une perspective plus large de réduction des inégalités sociales et des disparités entre les territoires.

Nos résultats militent ainsi en faveur de l'importance de soutenir la participation sociale en créant des environnements qui la facilitent. Compte tenu de l'abolition de programmes et de nouveaux mécanismes d'attribution, nous souhaitons attirer l'attention sur la nécessité de mieux comprendre les conséquences de ces transformations à l'échelle locale, et ce, particulièrement dans les petites communautés disposant de peu de ressources. Pour ce faire, les voix et les expériences vécues des citoyens concernés apparaissent riches pour inspirer l'action et alimenter des pistes de recherche. 


\section{RÉFÉRENCES}

Alberio, M. (2015). Les initiatives locales et les défis des acteurs du milieu face aux coupes et aux changements actuels. Organisations \& Territoires, 24(3), 53-60. https://doi.org/10.1522/revueot.v24i3.90

Bertaux, D. (2016). Le récit de vie (4e éd.). Paris, France : Armand Colin.

Birtch, T. (2017). Rural volunteerism: How well is the heart of community doing? Guelph, ON: Rural Ontario Institute. Repéré à www.ruralontarioinstitute.ca/uploads/userfiles/files/Rural\%20-Ontario\%20Foresight\%20Papers\%202017_ Volunteerism $\% 20$ and $\% 20$ Northern $\% 20$ Perspective.pdf

Boisvert, R. (2013). Réduction des inégalités sociales de santé : dispositions actantielles et intelligence collective au service du développement des communautés. Nowvelles pratiques sociales, 26(1), 197-214. https://doi.org/10.7202/1024988ar

Bonneau, M.-A. (2018). La participation sociale et ses retombées dans une perspective de santé et bien-être pour une communauté rurale québécoise (Mémoire de maitrise). Université Laval, Québec, QC. Repéré à https://corpus.ulaval.ca/jspui/ bitstream/20.500.11794/32807/1/34580.pdf

Bourque, D. et Favreau, L. (2003). Le développement des communautés et la santé publique au Québec. Service social, 50(1), 295-308. https://doi.org/10.7202/011352ar

Breviglieri, M. et Gaudet, S. (2014). Présentation : les arrières-scènes participatives et le lien ordinaire au politique. Lien social et Politiques, 71, 3-9. https://doi.org/10.7202/1024735ar

Falk, I. et Kilpatrick, S. (2000). What is social capital? A study of interaction in a rural community. Sociologia Ruralis, 40(1), 87-110. https://doi.org/10.1111/1467-9523.00133

Fédération québécoise des municipalités (FQM). (2015). Mémoire, Projet de loi no 28 : Loi concernant principalement la mise en ceuvre de certaines dispositions du discours sur le budget du 4 juin 2014 et visant le retour à l'équilibre budgétaire en 2015-2016, Québec, QC : FQM. Repéré à www.fqm.ca/wp-content/uploads/2015/02/memfqm-pl28-03022015.pdf

Gaudet, S. (2012). Lire les inégalités à travers les pratiques de participation sociale. SociologieS [En ligne], Débats, Penser les inégalités. Repéré à http://journals.openedition.org/sociologies/3874

Gaudet, S. et Turcotte, M. (2013). Sommes-nous égaux devant l'« injonction » à participer? Analyse des ressources et des opportunités au cours de la vie. Sociologie et sociétés, 45(1), 117-145. https://doi.org/10.7202/1016398ar

Godbout, J. T. et Caillé, A. (1992). L’esprit du don. Paris, France : La Découverte.

Gohier, C. (2004). De la démarcation entre critères d'ordre scientifique et d'ordre éthique en recherche interprétative. Recherches qualitatives, 24(1), 3-17. Repéré à www.recherche-qualitative.qc.ca/documents/files/revue/edition_reguliere/numero24/ 24gohier.pdf

Gravel, M.-A. (2017). Le bénévolat au Québec, de 2004 à 2013. Coup d'cil sociodémographique, 52. Québec, QC : Institut de la Statistique du Québec. Repéré à www.stat.gouv.qc.ca/statistiques/population-demographie/bulletins/coupdoeil-no52.pdf

Groupe de travail sur les communautés dévitalisées. (2010). Des communautés à revitaliser : un défi collectif pour le Québec. Québec, QC : Gouvernement du Québec. Repéré à www.mamh.gouv.qc.ca/fileadmin/publications/grands_dossiers/ municipalites_devitalisees/rapport_communautes_devitalisees.pdf

Hawe, P. et Shiell, A. (2000). Social capital and health promotion: A review. Social Science \& Medicine, 51(6), 871-885. https://doi.org/10.1016/S0277-9536(00)00067-8

Jean, B. (2006). Présentation - Le développement territorial : un nouveau regard sur les régions du Québec. Recherches sociographiques, 47(3), 465-474. https://doi.org/10.7202/014654ar

Jean, B. (2011). Territoires d'avenir: pour une sociologie de la ruralité. Québec, QC : PUQ.

Kenny, A., Hyett, N., Sawtell, J., Dickson-Swift, V., Farmer, J. et O’Meara, P. (2013). Community participation in rural health: A scoping review. BMC Health Services Research, 13(1), 64. https://doi.org/10.1186/1472-6963-13-64

Kilpatrick, S. (2009). Multi-level rural community engagement in health. Australian Journal of Rural Health, 17(1), 39-44. https://doi.org/10.1111/j.1440-1584.2008.01035.x

Klein, J.-L. (2012). L'économie sociale et solidaire et la lutte contre la pauvreté en milieu local : le cas du Québec. Revue internationale de l'économie sociale : Recma, 325, 65-77. https://doi.org/10.7202/-1017423ar 
Lachapelle, R. et Bourque, D. (2008). Les pratiques d'organisation communautaire en CSSS à l'épreuve des programmes de santé publique. Service social, 54(1), 23-39. https://doi.org/10.7202/018-341ar

Lévesque, J., Delisle, N.-A. et Leroux, R. (2002). La santé des communautés : perspectives pour la contribution de la santé publique au développement social et au développement des communautés. Montréal, QC : Institut national de santé publique du Québec. Repéré à www.inspq.qc.ca/pdf/publications/082_SanteCommunautes.pdf

Loignon, C., Dupéré, S., Godrie, B. et Leblanc, C. (2018). «Dés-élitiser » la recherche pour favoriser l'équité en santé : les recherches participatives avec des publics en situation de pauvreté en santé publique. Éthique publique : Revue internationale d'éthique sociétale et gouvernementale, 20(2). https://doi.org/10.4000/ethiquepublique.4058

Malet, J. (2009). Les associations, source de vitalité du milieu rural? Pour, 2, 97-102. https://doi.org/10.3917/pour.201.0097

Mercier, C., Bourque, D. et St-Germain, L. (2009). Participation citoyenne et développement des communautés au Québec : enjeux, défis et conditions d'actualisation. Gatineau, QC : UQO et ARUC-ISDC. Repéré à http://www.centrersa.qc.ca/pdf/ARUC-LSTG-PC-2009.pdf

Milligan, C. (2007). Geographies of voluntarism: Mapping the terrain. Geography Compass, 1(2), 183-199. https://doi.org/10.1111/j.1749-8198.2007.00008.x

Ministère des Affaires municipales, des Régions et de l'Occupation du territoire (MAMROT). (2013). Plan d'action en occupation et en vitalité des territoires du ministère des Affaires municipales, des Régions et de l'Occupation du territoire 2013-2016. Québec, QC : Gouvernement du Québec. Repéré à www.mamh.gouv.qc.ca/fileadmin/publications/occupation_territoire/plan_action_OVT.pdf

Parent, A.-A., Lachapelle, R., Bourque, D. et Jetté, C. (2016). Pratiques de développement territorial intégré. Orranisations \& Territoires, 25(2), 19-28. https://doi.org/10.1522/revueot.v25n2.311

Polèse, M. (2018). De politique régionale à développement local - Récit d'un succès québécois (ou presque) : jumeler équité territoriale et entrepreneurship local. Notes de recherche, no 2018-04. Québec, QC : Institut national de la recherche scientifique. Repéré à http:/ / espace.inrs.ca/id/eprint/7407

Putnam, R. D. (2000). Bowling alone: The collapse and revival of American community. New York, NY : Simon \& Schuster.

Québec. (2015). Loi concernant principalement la mise en ceuvre de certaines dispositions du discours sur le budget du 4 juin 2014 et visant le retour à l'équilibre budgétaire en 2015-2016 (RLRQ), [Québec], Éditeur officiel du Québec.

Reimer, B. (2006). The rural context of community development in Canada. Journal of Rural and Community Development, 1(2). Repéré à https://journals.brandonu.ca/jrcd/article/view/25/6

Richard, J., Bourque, D. et Lachapelle, R. (2017). Impacts de la transformation des politiques publiques sur le développement des territoires. Cahier no 17-03. Gatineau, QC : Chaire de recherche du Canada en organisation communautaire. Repéré à http://w4.uqo.ca/crcoc/Fichiers/cahiers/17-03_impact_transfo_pol_publique_sur_DT.pdf

Richardson, M. et Simard, P. (2016). Les effets de la Politique nationale de la ruralité du Québec sur la santé des ruraux et des communautés. Québec, QC : Fonds de recherche du Québec - Société et culture (FRQSC). Repéré à www.frqsc.gouv.qc.ca/ documents/11326/449012/PT_SimardP_resume_ruralite.pdf/5f3f6666-fde3-4cc5-84ec-ac8cf338eb37

Scalzo, D. (2016). Vers une plus grande marginalisation des municipalités rurales. Vie économique, $8(1)$. Repéré à www.eve.coop/?a=248

Simard, P., Parent, A.-A. et Richardson, M. (2018). La lutte à la pauvreté dans une perspective de développement des communautés : enjeux et défis dans un contexte en profonde transformation. Nowvelles pratiques sociales, 30(1). https://doi.org/10.7202/1051402ar

Skinner, M. W. et Joseph, A. E. (2011). Placing voluntarism within evolving spaces of care in ageing rural communities. GeoJournal, 76(2), 151-162. https://doi.org/10.1007/s10708-009-9283-8

Solidarité rurale du Québec. (2011). L'information régionale et communautaire, un des leviers de l'occupation des territoires : mémoire déposé au ministère de la Culture, des Communications et de la Condition féminine. Nicolet, QC : Solidarité rurale du Québec. Repéré à www.mcc.gouv.qc.ca/fileadmin/documents/consultation-publique/Trois-Rivieres/memoire\%20Solidarite\%20rurale.pdf

Statistique Canada. (2004). L'Enquête sociale générale : l'aperçu. Produit no 89F0115XIF au catalogue. Ottawa, ON : Statistique Canada. Repéré à www150.statcan.gc.ca/n1/fr/pub/89f0115x/89f0115x2004001-fra.pdf?st=POB1cles

Tremblay, P.-A. et Klein, J.-L. (1997). De l'appauvrissement des lieux à la reconstruction des communautés. Cabiers de recherche sociologique, 29, 103-118. https://doi.org/10.7202/1002678ar

Turcotte, M. (2005). L'engagement social et la participation à la vie communautaire : les populations des régions rurales et petites villes ont-elles véritablement l'avantage? Bulletin d'analyse : Régions rurales et petites villes du Canada, 6(4). Produit no 21-006-XIF au catalogue. 
Ottawa, ON : Statistique Canada. Repéré à www150.statcan.gc.ca/n1/fr/pub/21-006-x/21-006-x2005004-

fra.pd?st=K0u6wQOt

Vézina, M. et Compton S. (2012). Le bénévolat au Canada. Tendances sociales canadiennes, 93. Produit no 11-008-X au catalogue. Ottawa, ON : Statistique Canada. Repéré à www150.statcan.gc.ca/n1/fr/pub/11-008-x/2012001/article/11638-fra.pdf?st=mo4Cx3YE

Wallerstein, N. (2002). Empowerment to reduce health disparities. Scandinavian Journal of Public Health, 30(59 suppl), 72-77. https://doi.org/10.1177/14034948020300031201 